\title{
Understanding 2D atomic resolution imaging of the calcite surface in water by frequency modulation atomic force microscopy
}

\author{
John Tracey, ${ }^{1}$ Keisuke Miyazawa, ${ }^{2}$ Peter Spijker, ${ }^{1}$ Kazuki Miyata,${ }^{2}$ Bernhard Reischl, ${ }^{3}$ \\ Filippo Federici Canova, ${ }^{4}$ Andrew Rohl, ${ }^{3}$ Takeshi Fukuma, ${ }^{2,5}, *$ and Adam S. Foster ${ }^{1,2, \dagger}$ \\ ${ }^{1}$ COMP Centre of Excellence, Department of Applied Physics, \\ Aalto University, Helsinki FI-00076, Finland \\ ${ }^{2}$ Division of Electrical Engineering and Computer Science, \\ Kanazawa University, Kanazawa 920-1192, Japan \\ ${ }^{3}$ Curtin Institute for Computation, Nanochemistry Research Institute, \\ Department of Chemistry, Curtin University, Perth, WA 6845, Australia \\ ${ }^{4}$ Aalto Science Institute, Aalto University, Helsinki FI-00076, Finland \\ ${ }^{5}$ ACT-C, Japan Science and Technology Agency, Saitama 332-0012, Japan
}

(Dated: July 27, 2016)

\begin{abstract}
Frequency modulation Atomic Force Microscopy (FM-AFM) experiments were performed on the calcite (1014) surface in pure water, and a detailed analysis was made of the $2 \mathrm{D}$ images at a variety of frequency setpoints. We observed eight different contrast patterns that reproducibly appeared in different experiments and with different measurement parameters. We then performed systematic free energy calculations of the same system using atomistic molecular dynamics to obtain an effective force field for the tip-surface interaction. By using this force field in a virtual AFM simulation we found that each experimental contrast could be reproduced in our simulations by changing the setpoint, regardless of the experimental parameters. This approach offers a generic method for understanding the wide variety of contrast patterns seen on the calcite surface in water, and is generally applicable to AFM imaging in liquids.
\end{abstract}




\section{INTRODUCTION}

Frequency modulation Atomic Force Microscopy (FM-AFM) ${ }^{1}$ has become an increasingly powerful tool for studying materials in liquid environments with molecular resolution ${ }^{2}$. In particular, the ability to measure 3D force distributions in liquid offers unprecedented understanding of the hydration structures at ionic surfaces ${ }^{3-9}$. This capability encourages the application of FM-AFM to study processes where hydration plays a key role, such as biomolecular function ${ }^{10}$, crystal growth $^{11,12}$, soil wetting ${ }^{13}$ and catalytic reactions ${ }^{14}$.

Although FM-AFM in liquids has the potential to go beyond the level of detail offered in conventional spectroscopic techniques usually applied to study solid-liquid interfaces ${ }^{15-21}$, it also provides significant challenges in interpretation. This has motivated significant efforts to link experimental efforts to simulations, from simple models based on water density alone $^{22-24}$, to more complex modelling including a complete tip-surface system ${ }^{9,25-31}$. Most recently, a detailed comparison was made between experimental and simulated 3D force fields obtained in liquid on the calcite (10̄14) surface $^{9}$. This established a detailed understanding of the contrast mechanism, and provided strong evidence that the simulations captured the fundamental physical processes occurring. However, obtaining 3D forces in both experiment and theory is very time consuming, and for wider application it is also important to understand 2D images and how they relate to the imaging conditions $s^{3,5,7,8,32,33}$. In previous studies both analytical ${ }^{34-36}$ and full simulators ${ }^{37-43}$ have been used to calculate simulated images. The former lacks the electronics that describe the finite feedback of a AFM experiment and the latter generally only describe one particular experimental setup, and are not easily applicable to the system we study here.

In this work, we use the methods developed to understand 3D spectroscopy on calcite ${ }^{9}$, and apply them to understand a wide variety of $2 \mathrm{D}$ image contrast patterns. This provides an FM-AFM imaging reference for this benchmark surface, and also offers a template for general modelling and understanding $2 \mathrm{D}$ imaging contrasts in liquids. 


\section{METHODS}

\section{A. Experimental methods}

For the AFM experiments, we used a calcite(1014) (Crystal Base Co, Ltd.) substrate with a size of $5 \times 5 \times 2 \mathrm{~mm}^{3}$. The substrate was glued on a sample holder and cleaved by a razor blade. Immediately after the cleavage, we dropped $50 \mu \mathrm{l}$ Milli-Q water onto the sample surface. In the deposited water, we performed FM-AFM imaging in the constant frequency shift mode at room temperature. We used commercially available silicon cantilevers (AC55, Olympus), with a typical spring constant $k$, quality factor $Q$ and resonance frequency $f_{0}$ of $80 \mathrm{~N} / \mathrm{m}, 10$ and $1.5 \mathrm{MHz}$, respectively. In order to improve the stability and reproducibility in the atomic-scale imaging, the tip was coated with $30 \mathrm{~nm}$ silicon film by a dc sputter coater $(\mathrm{K} 575 \mathrm{XD}, \text { Emitech })^{44}$. The oscillation amplitude is typically chosen to optimise the image quality. Theoretically, the maximum signal-to-noise ratio is achieved with an amplitude similar to the decay length of the interaction force to be detected ${ }^{45}$. This corresponds to 0.1$0.5 \mathrm{~nm}$ in the case of hydration force. In fact, in our experiments, we found that amplitude values within this range provide the best image quality. Even though the existence of the oscillatory force profile sometimes leads to the instabilities in the tip-sample distance regulation, it is a convention within FM-AFM in liquids to accept such instabilities to achieve the best image quality using a small amplitude.

We used two different home-build AFM systems. Both are equipped with an ultra low-noise cantilever deflection sensor ${ }^{46,47}$ and a high-stability photothermal excitation system $^{48,49}$. One is for standard-speed imaging ( $\sim 60 \mathrm{~s} /$ frame), while the other is for highspeed imaging $(\sim 2 \mathrm{~s} / \text { frame })^{50}$. For the standard system, we used a commercially available AFM controller (ARC2, Asylum Research) and a cantilever oscillation controller (OC4, SPECS). For the high-speed system, we used a custom-built AFM controller with a field programmable gate array (FPGA) chip (Vertex-5, Xilinx), where we implemented high-speed scanning control, data recording and cantilever oscillation control functions.

\section{B. Simulation methods}

The simulation protocol for computing the tip-surface interactions and free energy is very similar to that already introduced previously ${ }^{9,31}$, and here we only give a brief overview and 
highlight any differences. The atomistic model of the system consisted of a slab of calcite, 7 layers thick, exposing the (1014) surface along $z$ and a 160 atom calcite nanocluster terminating in a $\mathrm{Ca}^{2+}$ ion, modeling the AFM tip apex. The latter is a reasonable tip model, as in experiments the silicon tip almost always contacts the surface before imaging and, thus, becomes covered with calcite ${ }^{9}$. The whole system was fully solvated by $\mathrm{SPC} / \mathrm{Fw}$ water molecules in a rectangular simulation box with periodic boundaries, measuring 4.06 $\times 3.95 \times 9.77 \mathrm{~nm}^{3}$. Atomistic interactions are described by the force field developed by Raiteri et al. ${ }^{12}$ We used the GROMACS simulation suite versions 4.5 and $4.6^{51}$ to carry out molecular dynamics simulations, and umbrella sampling to obtain free energy profiles as a function of the tip-surface distance, over $8 \times 4$ points on a calcite surface unit cell. A 3D map of the force acting on the tip was obtained from the derivative of these free energy profiles.

In order to obtain simulated 2D images we used the Python Virtual Atomic Force Microscope (PyVAFM) ${ }^{52}$ to simulate the full scan of the surface by numerically solving the equation of motion of the cantilever with parameters $k, f_{0}, Q$, oscillating with an amplitude $A$ in the $3 \mathrm{D}$ force field obtained from the atomistic simulation. The PyVAFM is a simulation package which allows the reproduction of any AFM experiment using a modular simulation, where each individual module (known as a circuit) describes a realistic component or process, e.g., a low pass filter. By connecting these circuits one can match the experimental set-up as close as possible and reproduce images from any experimental set up or operational mode. In Section II A two different experimental AFM setups (standard and fast) are described, which have besides different imaging speeds also different unique cantilever parameters. For the fast AFM setup these are $f_{0}=1.553 \mathrm{MHz}, k=130 \mathrm{~N} / \mathrm{m}, A=0.13 \mathrm{~nm}$ and $Q=10$, whereas the standard AFM setup has $f_{0}=1.29 \mathrm{MHz}, k=75.1 \mathrm{~N} / \mathrm{m}, A=0.21 \mathrm{~nm}$ or $0.23 \mathrm{~nm}$ and $Q=11.1$. In order to obtain simulated 2D images, several hundred frequency setpoints, effectively corresponding to different average tip-surface distances, were simulated and compared to experimental results in order to find the best match. Please note that the average tip height in this work is defined by the distance between tip apex and sample atoms, which is $1.86 \mathrm{~nm}$ smaller than the centre of mass distance used in the previous comparison of $3 \mathrm{D}$ data $^{9}$. Furthermore, since we did not observe any significant differences in the approach and retract curves in experiments, the force field we are using in simulations is fully conservative, and we are not considering any dissipative effects ${ }^{53-56}$. 


\section{Constant frequency imaging in liquids}

Above different crystal sites (e.g., above $\mathrm{Ca}$ or $\mathrm{CO}_{3}$ ), different frequency shift responses are observed, see Fig. 1a where three representative responses as obtained from the atomistic free energy simulations are depicted. Now, for example, if we assume a frequency shift setpoint of $1 \mathrm{kHz}$ (the horizontal blue line in Fig. 1a) and if we then approach the surface (i.e., coming from the right in Fig. 1a) we can see that we can intersect the frequency shift response curves at different heights (e.g., for both $\mathrm{CO}_{3}$ sites at around $0.58,0.40,0.35$ or $0.29 \mathrm{~nm}$ ). Thus, there are different heights at which the setpoint can be found above a given site, which is in contrast to nearly all measurements in vacuum. This has an important effect on an approaching cantilever, as is explained in Fig. 1b. In this figure, different $\Delta f$ curves above various sites (numbered from 1 through 9, with their location depicted in Fig. 1c) are shown together with the piezo height over a scan line. At the initial piezo position over a Ca site (1 in 1b) the tip is around $0.8 \mathrm{~nm}$ from the surface, it then scans laterally and finds a similar $\Delta f$ at position 2 . As it passes the protruding oxygen of the carbonate group (3), it descends to below $0.6 \mathrm{~nm}$ to find the same $\Delta f$. When keeping scanning, despite being over an effectively equivalent Ca site (4), it descends to find a $\Delta f$ much closer to the surface, as in this case the setpoint can be found at several heights (see Fig. 1a) and the magnitude of the $\Delta f$ relative to the setpoint determines the direction of search (a larger $\Delta f$ than the setpoint causes descent in this case). Once stabilised in this regime, the scanline from 4-6 is repeated from 7-9, with the only differences due to the thermodynamically caused variations in the carbonate configurations (see also Fig. 1d for a side view). In general, this effect can be quite drastic, resulting in significant height jumps (as seen while going from 2 to 4). Hence when simulating images, care must be taken that enough of the image has been scanned in order to insure the piezo height is low enough to obtain a periodic response - just as in experiments.

In this context it is import to point out that when naively applying an analytical formulation, when converting a $\Delta f$ into an image, and searching for where the frequency change $(\Delta f)$ is equal to the setpoint may, in the case of constant frequency experiments in liquids, yield incorrect results, due to the possible different heights for a given setpoint. Consequently, it is important in the simulations to take into account how the electronics in the given experiment react to the changes in $\Delta f$. For example, in the experiment when the $\Delta f$ 
(a)

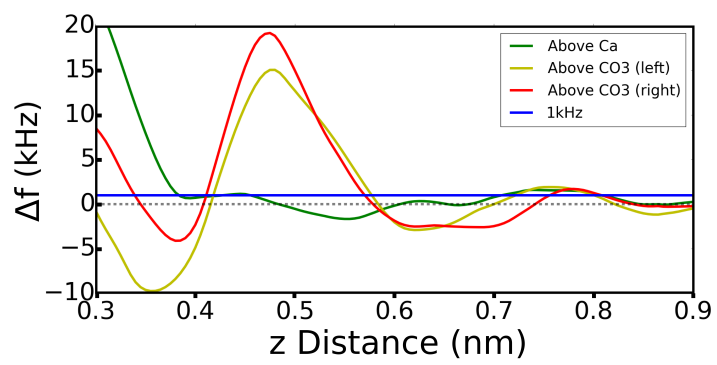

(b)

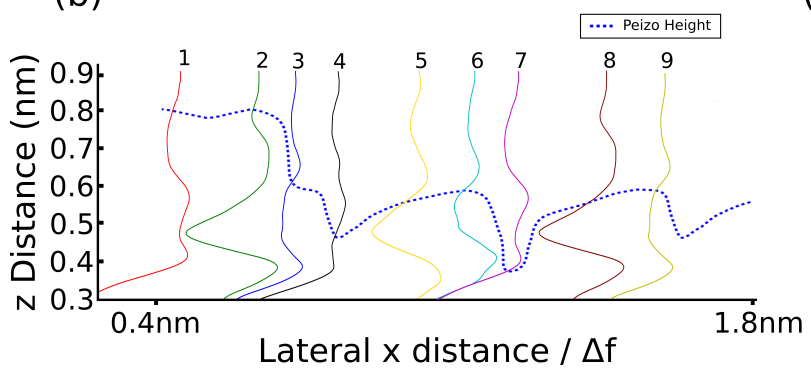

(c)

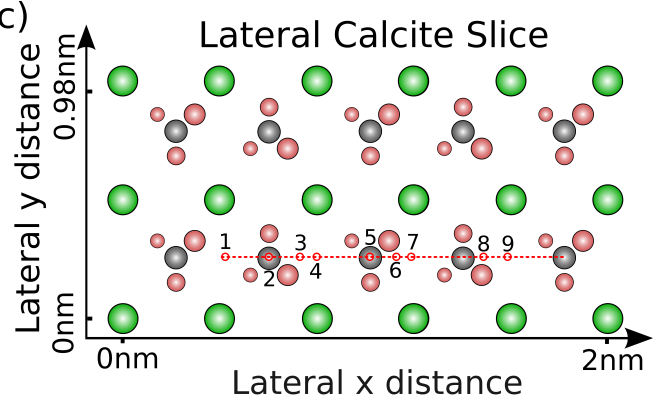

(d)

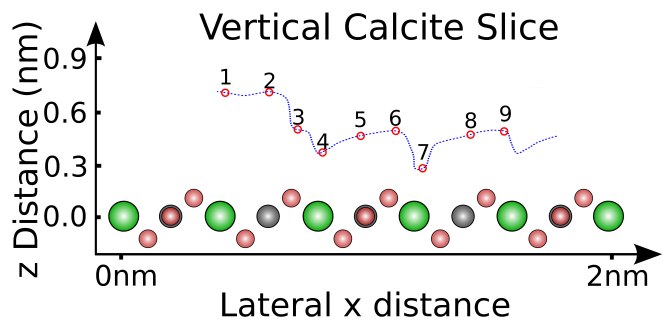

FIG. 1: In (a) the $\Delta f$ curves above a Ca site (green), both inequivalent $\mathrm{CO}_{3}$ sites (yellow and red) and a line designating frequency shift setpoint of $\Delta f=1 \mathrm{kHz}$ (blue) are shown. In (b) the change in piezo height (dotted line) required to obtain a constant frequency shift of $1 \mathrm{kHz}$, and the associated $\Delta f$ curves above various sites are depicted. The numbers correspond with the different sites and are indicated (together with the scan line) on the calcite (1014) surface in (c). In (d) the piezo height is show in a side view of the surface as it moves across the different sites.

is lower than the setpoint the tip is brought closer to the surface and the tip is moved further away when the $\Delta f$ is larger than the setpoint. As a result of this it is possible for a given site that the cantilever can obtain the setpoint at a given height, but on reaching a periodically similar site, finds itself at a lower $z$ position despite the site being identical. Hence it can be concluded that the height of the tip at any given setpoint depends on the height scanning a particular site. The PyVAFM automatically recovers the correct behaviour in real experiments, as it is modeling all the electronics, but analytical approaches ${ }^{57,58}$ must be modified to account for this multiple height for a given setpoint effect. 


\section{IMAGE RECOGNITION ALGORITHM}

In the next section we compare our theoretical images with the experimental results. In order to aid us we developed an image recognition algorithm, which compares an experimental image to all our simulated images. The basis of the algorithm is to analyse and compare the 2D image Fourier spectrum. Taking the Fourier transform yields several advantages: atomically resolved images tend to exhibit high levels of periodicity resulting in strong peaks in the final spectrum; the transforms are phase invariant, which is vital since atomic sites cannot be explicitly identified from experimental images; additionally the transforms are insensitive to scale, so that an image that contains one unit cell, yields the same spatial frequencies as an image that contains two or more unit cells. A similarity "score" is obtained by taking each line of the Fourier transform of the image and comparing it directly to the corresponding line in the reference image. This is repeated for each line and an average difference between each line is computed. In addition to this score we also examine the amplitude difference between the two images to obtain an amplitude matching score. In the case of constant frequency experiments, we compare the average change in height of the z-piezo in both the reference experimental image and the simulated images. A final score is computed by summing both scores, with a weighting of $90 \%$ towards pattern matching and 10\% towards amplitude matching. Out of the 27 computed images 11 of the chosen images were within the top $5 \%$ of scores, 7 within the top 10\%, 3 within the top $15 \%, 3$ within the top $25 \%$ and 3 within the top $35 \%$.

\section{RESULTS AND DISCUSSION}

In Fig. 2 we present a set of eight experimental contrast patterns that we consider distinct and reproducible across several experiments. Most of the patterns seen are associated with a particular set of measurement parameters, i.e., fast or standard, but there are often similar images in both setups and, for instance, contrast pattern 2 is very clearly seen in both. The raw data presented in Fig. 2 has considerable noise and is not corrected for drift. Hence it has been post-processed by applying an averaging filter over the image and correcting for thermal drift to remove any distortion. Finally, by applying a pattern matching algorithm 
(a) Pattern 1

Fast $(\Delta f=1.9 \mathrm{kHz}, A=0.13 \mathrm{~nm})$

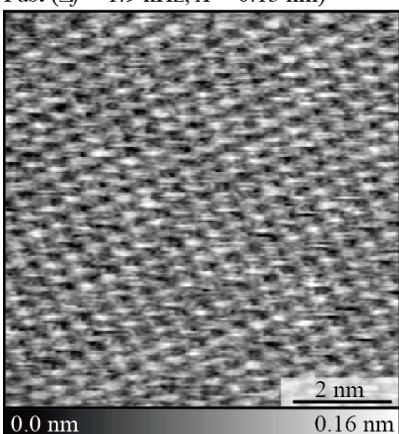

(e) Pattern 5

Fast $(\Delta f=1.6 \mathrm{kHz}, A=0.13 \mathrm{~nm})$

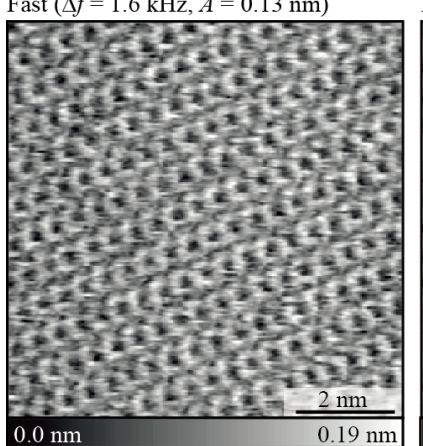

(b) Pattern 2

(i) Fast $(\Delta f=2.1 \mathrm{kHz}, A=0.13 \mathrm{~nm})$

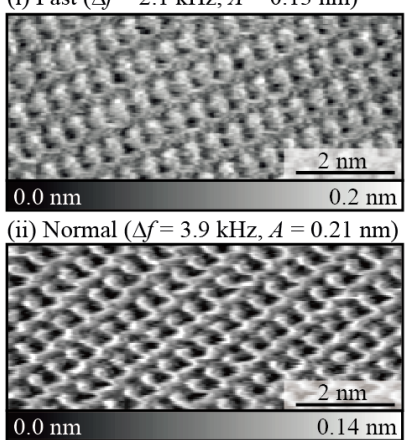

(b) Pattern 6

Normal $(\Delta f=5.9 \mathrm{kHz}, A=0.23 \mathrm{~mm})$

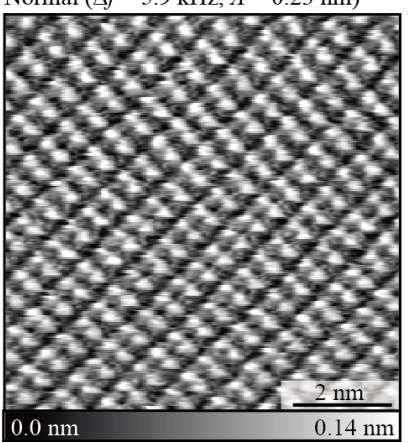

(c) Pattern 3

Fast $(\Delta f=2.1 \mathrm{kHz}, A=0.13 \mathrm{~nm})$

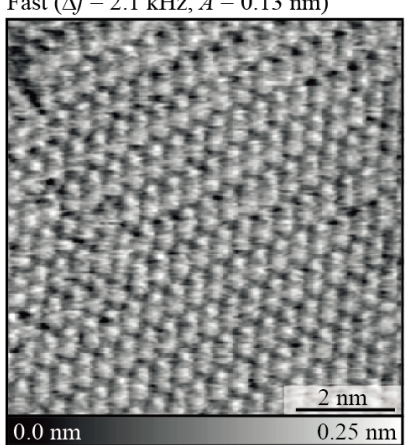

(c) Pattern 7

$(\mathrm{Hz} A=0.23 \mathrm{~nm})$

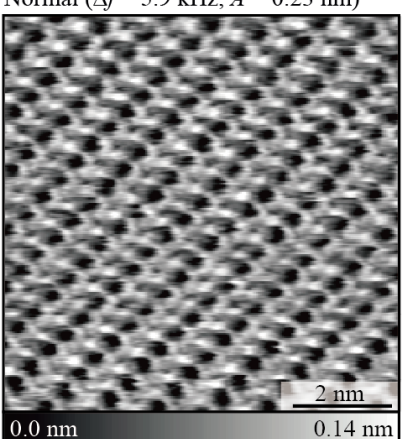

(d) Pattern 4

Fast $(\Delta f=1.6 \mathrm{kHz}, A=0.13 \mathrm{~nm})$

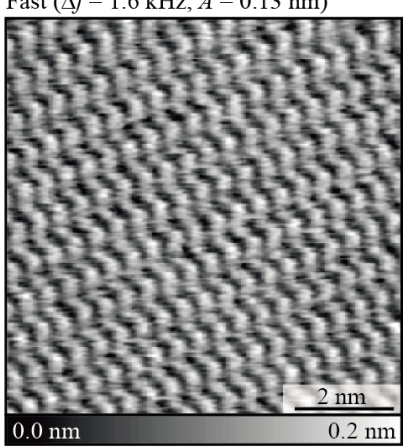

(b) Pattern 8

$\operatorname{Normal}(\Delta f=10.2 \mathrm{kHz}, A=0.23 \mathrm{~nm})$

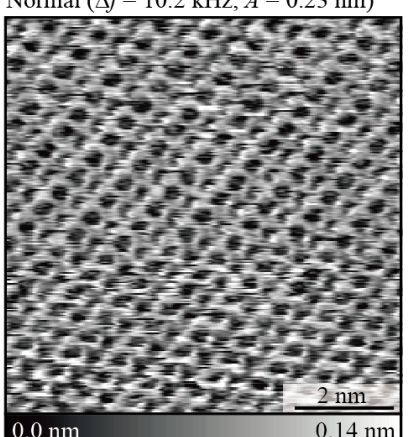

FIG. 2: The eight distinct and reproducible contrast patterns observed in experiments, for different frequency shift setpoint $\Delta f$ and oscillation amplitudes $A$.

it is possible to further enhance the image, taking into account the whole image to provide an averaged image slightly larger than one unit cell. These enhanced data images, as well as the simulated images, are presented in Figs. 3 and 4.

The simulated images were selected with assistance of the algorithm discussed in section III from hundreds of images at different setpoints to find the best matches, regardless of the parameter set used. However, the simulated data did not show large variations in the contrast patterns according to the setup, as in experiments, and in order to emphasize this, Figs. 3 and 4 show simulated images for each contrast pattern with three different virtual AFM setups, representing the fast and two standard parameter sets. In general, the dominating factor in the contrast pattern observed is the average height of the tip, as this determines the forces experienced by the tip, and the observed contrasts are ordered 


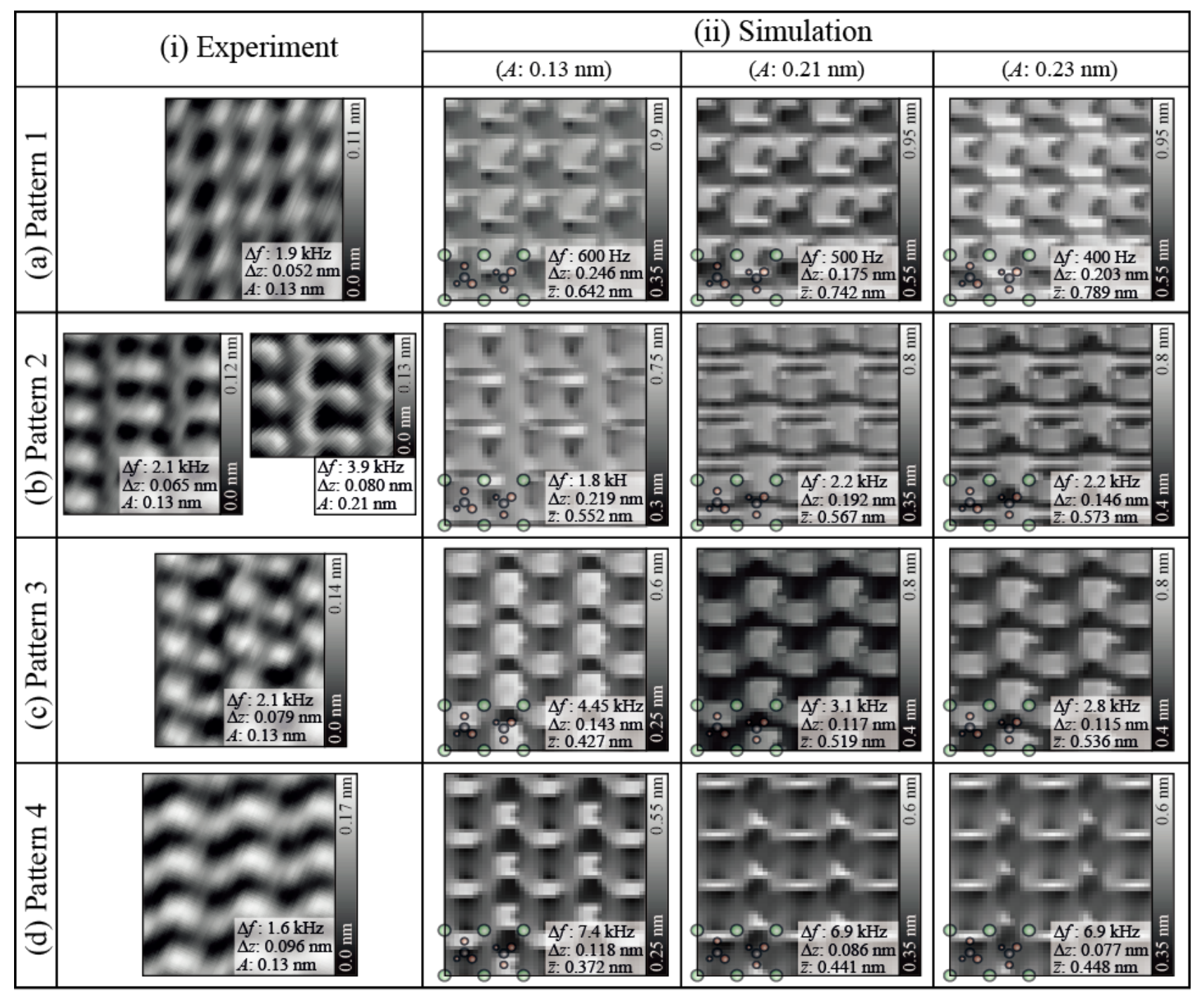

FIG. 3: A comparison of the characteristic experimental images repeatedly observed and corresponding simulated images for contrast patterns 1 to $4 . \Delta f$ is frequency shift setpoint, $A$ is amplitude, $\Delta z$ is the average contrast in topography and $\bar{z}$ is the average tip height in simulations across an image.

with respect to the predicted average height - from over $0.7 \mathrm{~nm}$ in contrast pattern 1 to less than $0.3 \mathrm{~nm}$ in contrast pattern 8 . While the simulated and experimental 2D images show good qualitative agreement, some quantitative differences exist, reflecting the fact that we do not have exactly the same tip as in experiments - in fact we use a highly idealized model of the experimental tip in our simulations. In light of this, it is even more remarkable that simulations appear to recover all the observed contrast patterns reasonably well with a single tip and force field, which is rare, even for imaging insulators in vacuum ${ }^{59,60}$. Looking 


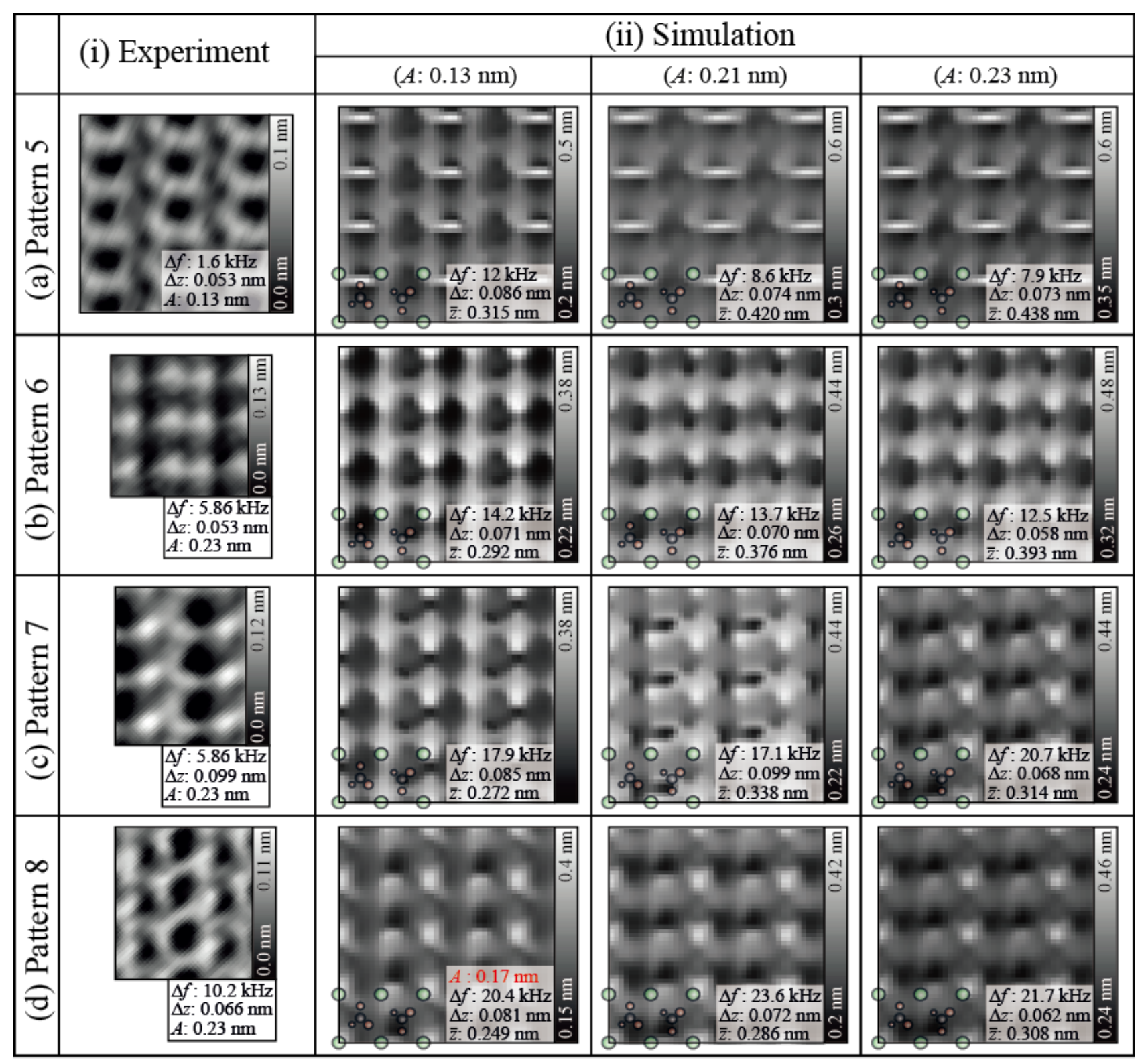

FIG. 4: A comparison of the characteristic experimental images repeatedly observed and corresponding simulated images for contrast patterns 5 to $8 . \Delta f$ is frequency shift setpoint, $A$ is amplitude, $\Delta z$ is the average contrast in topography and $\bar{z}$ is the average tip height in simulations across an image.

in detail, some contrast patterns are better reproduced by simulations, in particular 3, 4, 5, 6, while the others are somewhat more sensitive to the parameters. For example, in contrast pattern 2, the fast AFM simulated image shows the line and spot contrast seen in experiments more clearly than the other setups. It is likely no coincidence that agreement 
is less striking in the images at long-range (Fig. 3a,b) and close-range (Fig. 4c,d). At longrange, the forces are small and quite similar, and very small changes in the setpoint can cause large changes in the contrast (see Fig. 5). Closer to the surface, the forces become very large and depend strongly the exact nature of the tip and parameters used. In terms of a comparison of measured and simulated average contrast in topography ( $\Delta z$ in Figs. 3 and 4) the comparison also follows this trend to a degree, with the poorest agreement for contrast patterns 1 and 2 (simulations about 2-5 times larger), and very good agreement for all the other contrast patterns - confirming that the simulations are qualitatively capturing the site-dependent interactions.

Further analysis of the contrast patterns can be made by looking in detail at the relationship between water density, free energy, tip-sample force, gradient of the force and frequency shift. Fig. 5 shows this comparison over one $\mathrm{Ca}$ and one $\mathrm{CO}_{3}$ surface site. Here, the water density is calculated without the nanocluster tip, hence, it only shows the position of the hydration layers above the surface. Although the link between water density and free energy in the full tip-sample system is complex, the equilibrium hydration layer structure is reflected in the measured forces ${ }^{9}$, and in certain cases can be used as the basis of simpler modelling approaches ${ }^{22,23,31}$. This can be seen directly from the positions of the hydration layers in Figs. 5a and 5b, and the corresponding peaks in the other plots. The correlation varies across the different signals, but it is present in all channels.

Interestingly, the commonly used assumption that the frequency shift corresponds to the force gradient in AFM imaging ${ }^{1}$ appears not to hold in Fig. 5. This is because we must take into consideration that the cantilever is oscillating with some finite amplitude and samples the force over a range of tip-surface distances. This process is further complicated by the fact the velocity of the cantilever is not constant during the oscillation cycle, hence the forces felt at the turning points of the oscillation contribute more to the final $\Delta f$ signal. To illustrate this, in Fig. 6 we have plotted the oscillation range of the tip over the the force and frequency shift curves (calculated using our PyVAFM see section II B).

It is immediately evident that imaging at long-range, in the red region, will provide a fairly smooth average, as both extremes sample relatively similar interactions to the midpoint, i.e., the average distance at that setpoint. However, at close-range, in the blue region and also in mid-range (yellow) over the Ca site we see that there is significant differences in the interaction at the turning points, and the midpoint is not at all representative of the 
(a)

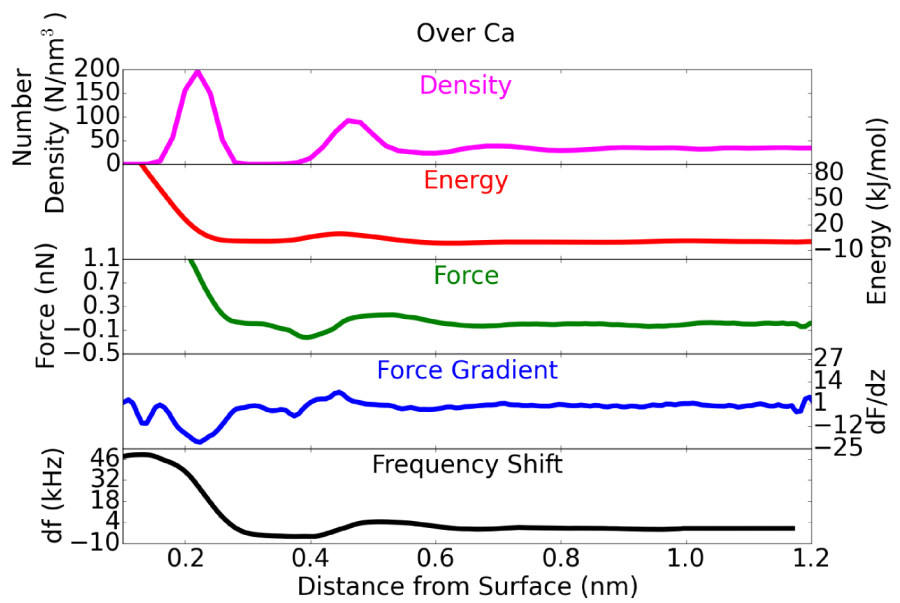

(b)

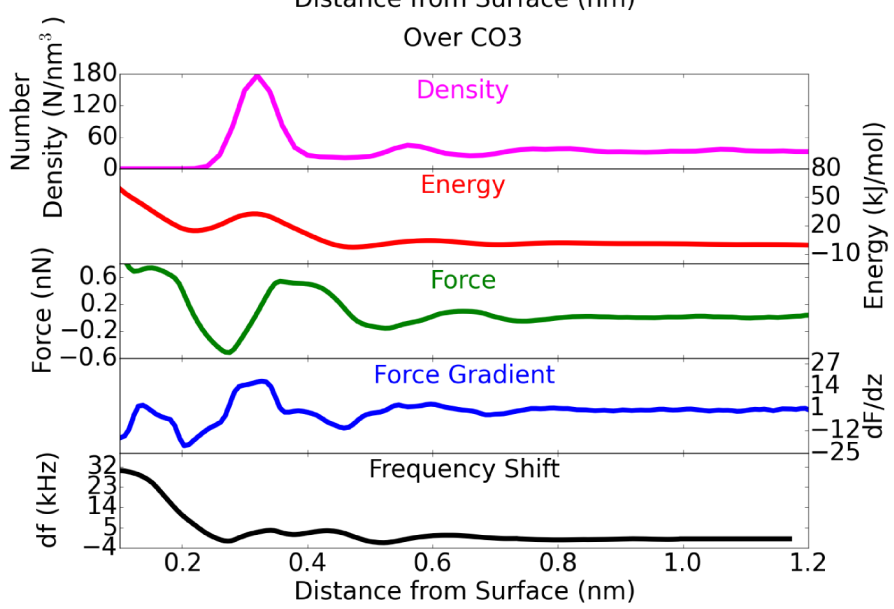

FIG. 5: The water density, free energy, tip-sample force, force gradient and simulated frequency shift (plotted with respect to lowest turning point) for sites above a (a) $\mathrm{Ca}$ and (b) $\mathrm{CO}_{3}$ site with $f_{0}=1.55 \mathrm{Mhz}, Q=10, k=130 \mathrm{~N} / \mathrm{m}, A=0.13 \mathrm{~nm}$.

interactions experienced along the whole oscillation.

If we reflect on the previous discussion of the quality of agreement between experimental and simulated images, Fig. 6 also provides an obvious explanation of why the amplitude has the largest effect on where these contrasts appear - at close approach changing the amplitude has a very large effect on the interactions sampled. As an example of this, the amplitude of the fast AFM simulation setup in contrast 8 was slightly increased to $0.17 \mathrm{~nm}$ (see Fig. $4 \mathrm{~d}$ ) allowing us to get a higher setpoint (hence also moving the tip closer to the surface), but still providing similar contrast to the other setups. Ultimately, this leads to an issue that 
in simulations the tip cannot be brought close enough (without crashing) to observe a given contrast. This is demonstrated in contrast pattern 8 in Fig. $4 \mathrm{~d}$ for $A=0.23 \mathrm{~nm}$, where it was impossible to get stable imaging at a closer average height $\bar{z}$ than $0.32 \mathrm{~nm}$ as the blue region in Fig. 6 is at the limit of the simulated data (even using smaller, unphysical amplitudes doesn't help much due to the rapid force changes in the region).
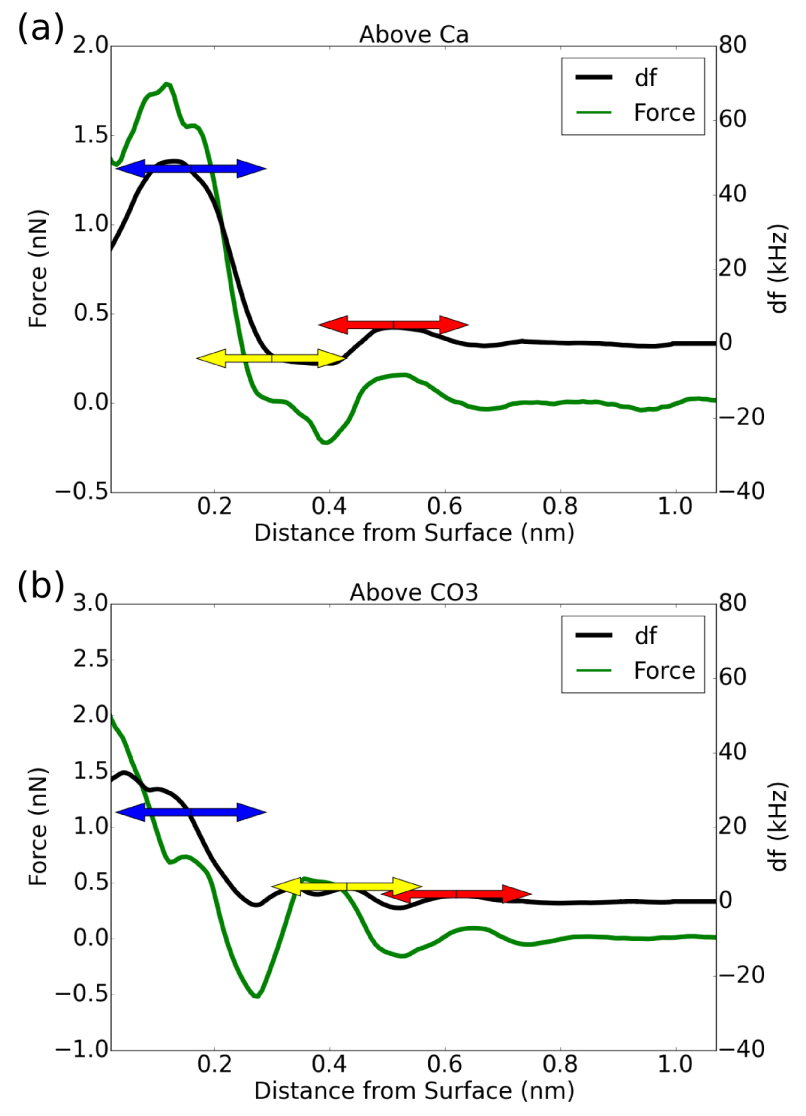

FIG. 6: Schematic example oscillations of the cantilever at long (red), medium (yellow) and close (blue) approach above (a) $\mathrm{Ca}$ and (b) $\mathrm{CO}_{3}$ sites overlaid on the simulated force and frequency shift (plotted with respect to lowest turning point), for a cantilever with parameters $f_{0}=1.55 \mathrm{Mhz}$, $Q=10, k=130 \mathrm{~N} / \mathrm{m}$, and $A=0.13 \mathrm{~nm}$.

\section{CONCLUSIONS}

In conclusion, a comparison of experimental and simulated 2D AFM images shows that all the characteristic contrasts seen in experiments can be understood in terms of the frequency setpoint (i.e., average tip-surface distance) and amplitude (i.e., interaction range sampled 
during an oscillation). This acts as a useful reference for future studies on this benchmark surface, in particular as deviations from these contrast patterns highlight systems where, for example, the tip, solvated ions or surface-induced processes are strongly changing the imaging conditions from the standard established here.

Within this study only one tip has been considered, and although the tip clearly plays a role in the absolute nature of 2D imaging, we can still obtain reasonable agreement between experimental and simulated images. Although tip preparation in all experiments was formally the same, the history of contact with the surface and reactions in the solvent must cause differences in tip chemistry and structure. Perhaps these are reflected in images at long- and close-range, and further studies could explore the tip-dependence in detail for a variety of plausible tip models, and also including experiments using functionalized tips. Control of the tip apex has been a revolution in low temperature, ultra high vacuum AFM imaging ${ }^{61}$, and anything approaching this in liquids would represent enormous progress.

Although this work has focused on interpretation of FM-AFM, the general conclusions are equally applicable to Amplitude Modulated $\mathrm{AFM}^{62}$, a parallel technique widely used in biomolecular studies, but also recently used to study ionic surfaces in high resolution ${ }^{63-67}$. The differences are mainly related to the experimental setup and procedure, and we plan to perform a systematic comparison of the two AFM imaging modes for calcite in liquid in the future.

\section{Acknowledgments}

This work was supported by KAKENHI (25706023), Japan Society for the Promotion of Science; ACT-C, Japan Science and Technology Agency; Kanazawa University CHOZEN Project; the Academy of Finland through its Centres of Excellence Program (project no. 251748). Development of the virtual AFM was supported by the EU through grant PAMS (seventh framework program GA 610446). B.R. has been supported by the Australian Research Council (DP140101776). We acknowledge use of the supercomputer resources of the Finnish CSC-IT Center for Science.

\footnotetext{
* Electronic address: fukuma@staff.kanazawa-u.ac.jp
} 
$\dagger$ Electronic address: adam.foster@aalto.fi

1 F. Giessibl, Rev. Mod. Phys. 75, 949 (2003).

2 S. Morita, F. J. Giessibl, E. Meyer, and R. Wiesendanger, eds., Noncontact Atomic Force Microscopy, NanoScience and Technology (Springer International Publishing, Cham, 2015), ISBN 978-3-319-15588-3.

3 S. Rode, N. Oyabu, K. Kobayashi, H. Yamada, and A. Kuehnle, Langmuir 25, 2850 (2009).

4 T. Fukuma, Y. Ueda, S. Yoshioka, and H. Asakawa, Phys. Rev. Lett. 104, 016101 (2010).

5 S. Rode, R. Hölscher, S. Sanna, S. Klassen, K. Kobayashi, H. Yamada, W. Schmidt, and A. Kühnle, Physical Review B 86, 075468 (2012).

${ }^{6}$ H. Asakawa, S. Yoshioka, K.-i. Nishimura, and T. Fukuma, ACS Nano 6, 9013 (2012).

7 K. Kobayashi, N. Oyabu, K. Kimura, S. Ido, K. Suzuki, T. Imai, K. Tagami, M. Tsukada, and H. Yamada, J. Chem. Phys. 138, 184704 (2013).

8 Y. Araki, K. Tsukamoto, R. Takagi, T. Miyashita, N. Oyabu, K. Kobayashi, and H. Yamada, Crystal Growth \& Design p. 62546260 (2014).

9 T. Fukuma, B. Reischl, P. Spijker, F. F. Canova, K. Miyazawa, and A. S. Foster, Phys. Rev. B 92, $155412(2015)$.

10 J. Ostmeyer, S. Chakrapani, A. C. Pan, E. Perozo, and B. Roux, Nature 501, 121 (2013).

11 H. H. Teng, P. M. Dove, and J. J. De Yoreo, Geochim. Cosmochim. Act. 64, 2255 (2000).

12 P. Raiteri and J. D. Gale, J. Am. Chem. Soc. 132, 17623 (2010).

13 P.-Y. Meslin, O. Gasnault, O. Forni, S. Schröder, A. Cousin, G. Berger, S. M. Clegg, J. Lasue, S. Maurice, V. Sautter, et al., Science 341, 1238670 (2013).

14 G. Dong, P. Teo, Z. K. Wickens, and R. H. Grubbs, Science 333, 1609 (2011).

15 K. B. Eisenthal, Chem. Rev. 96, 1343 (1996).

16 L. Cheng, P. Fenter, K. L. Nagy, M. L. Schlegel, and N. C. Sturchio, Phys. Rev. Lett. 87, $156103(2001)$.

17 B. Bagchi, Chem. Rev. 105, 3197 (2005).

18 S. K. Pal and A. H. Zewail, Chem. Rev. 104, 2099 (2004).

19 M. E. Johnson, C. Malardier-Jugroot, R. K. Murarka, and T. Head-Gordon, J. Phys. Chem. B 113, $4082(2009)$.

20 B. Jana, S. Pal, and B. Bagchi, J. Chem. Sci. 124, 317 (2012).

21 P. Fenter, S. Kerisit, P. Raiteri, and J. D. Gale, J. Phys. Chem. C 117, 5028 (2013). 
22 M. Watkins and B. Reischl, J. Chem. Phys. 138, 154703 (2013).

23 K.-I. Amano, K. Suzuki, T. Fukuma, O. Takahashi, and H. Onishi, J. Chem. Phys. 139, 224710 (2013).

24 P. Spijker, T. Hiasa, T. Musso, R. Nishioka, H. Onishi, and A. S. Foster, J. Phys. Chem. C 118, 2058 (2014).

25 M. Watkins and A. L. Shluger, Phys. Rev. Lett. 105, 196101 (2010).

26 M. Harada and M. Tsukada, Phys. Rev. B 82, 035414 (2010).

27 M. Tsukada, N. Watanabe, M. Harada, and K. Tagami, J. Vac. Sci. Technol., B: Microelectron. Nanometer Struct.-Process., Meas., Phenom. 28, C4C1 (2010).

28 M. Watkins, M. L. Berkowitz, and A. L. Shluger, Phys. Chem. Chem. Phys. 13, 12584 (2011).

29 D. Argyris, P. D. Ashby, and A. Striolo, ACS Nano 5, 2215 (2011).

30 D. Argyris, A. Phan, A. Striolo, and P. D. Ashby, J. Phys. Chem. C 117, 10433 (2013).

31 B. Reischl, M. Watkins, and A. S. Foster, J. Chem. Theory Comput. 9, 600 (2013).

32 F. Ohnesorge and G. Binnig, Science 260, 1451 (1993).

33 H. Imada, K. Kimura, and H. Onishi, Langmuir 29, 10744 (2013).

34 F. J. Giessibl, Physical Review B 56, 16010 (1997).

35 A. Livshits, A. Shluger, A. Rohl, and A. Foster, Physical Review B 59, 2436 (1999).

36 J. E. Sader and S. P. Jarvis, Applied Physics Letters 84, 1801 (2004), URL http://link.aip. org/link/?APPLAB/84/1801/1.

37 G. Couturier, J. P. Aime, J. Salardenne, R. Boisgard, A. Gourdon, and S. Gauthier, Applied Physics A Materials Science \& Processing 72, S47 (2001).

38 G. Couturier, J. P. Aim 'e, J. Salardenne, and R. Boisgard, The European Physical Journal Applied Physics 15, 141 (2001).

39 J. Polesel-Maris and S. Gauthier, Journal of Applied Physics 97, 044902 (2005).

40 L. Nony, A. Baratoff, D. Schär, O. Pfeiffer, A. Wetzel, and E. Meyer, Physical Review B 74, 235439 (2006), URL http://scitation.aip.org/getabs/servlet/GetabsServlet? prog=normal\&id=PRBMD0000074000023235439000001\&idtype=cvips\&gifs=yes.

41 M. Watkins, T. Trevethan, A. Shluger, and L. Kantorovich, Physical Review B 76, 245421 (2007).

42 T. Trevethan, L. Kantorovich, J. Polesel-Maris, S. Gauthier, and A. Shluger, Phys. Rev. B 76, 085414 (2007), URL http://link.aps.org/doi/10.1103/PhysRevB.76.085414. 
43 T. Trevethan, L. Kantorovich, J. Polesel-Maris, and S. Gauthier, Nanotechnology 18, 084017 (2007).

44 S. M. R. Akrami, H. Nakayachi, T. Watanabe-Nakayama, H. Asakawa, and T. Fukuma, Nanotechnology 25, 455701 (2014).

45 F. J. Giessibl, H. Bielefeldt, S. Hembacher, and J. Mannhart, Appl. Surf. Sci. 140, 352 (1999).

46 T. Fukuma, M. Kimura, K. Kobayashi, K. Matsushige, and H. Yamada, Rev. Sci. Instrum. 76, $053704(2005)$.

47 T. Fukuma and S. P. Jarvis, Rev. Sci. Instrum. 77, 043701 (2006).

48 T. Fukuma, Rev. Sci. Instrum. 80, 023707 (2009).

49 T. Fukuma, K. Onishi, N. Kobayashi, A. Matsuki, and H. Asakawa, Nanotechnology 23, 135706 (2012).

50 K. Miyata, H. Asakawa, and T. Fukuma, Appl. Phys. Lett. 103, 203104 (2013).

51 D. Van Der Spoel, E. Lindahl, B. Hess, G. Groenhof, A. E. Mark, and H. J. C. Berendsen, J. Comput. Chem. 26, 1701 (2005).

52 J. Tracey, F. Federici Canova, O. Keisanen, D. Z. Gao, P. Spijker, B. Reischl, and A. S. Foster, Comput. Phys. Commun. 196, 429 (2015).

53 F. Federici Canova, Nanotechnology 22, 045702 (2011).

54 S. Kawai, F. F. Canova, T. Glatzel, A. S. Foster, and E. Meyer, Phys. Rev. B 84, 115415 (2011).

55 F. Federici Canova, S. Kawai, C. de Capitani, K.-i. Kan'no, T. Glatzel, B. Such, A. S. Foster, and E. Meyer, Phys. Rev. Lett. 110, 203203 (2013).

56 S. Belikov, I. Yermolenko, and S. Magonov, P. Amer. Contr. Conf. p. 3485 (2015).

57 W. Hofer, A. Foster, and A. Shluger, Rev. Mod. Phys. 75, 1287 (2003).

58 A. Sebastian, A. Gannepalli, and M. V. Salapaka, Proceedings of the American Control Conference pp. 2499-2504 (2004).

59 R. Bechstein, C. Gonzalez, J. Schuette, P. Jelinek, R. Perez, and A. Kuehnle, Nanotechnology 20, 505703 (2009).

60 C. Barth, A. S. Foster, C. R. Henry, and A. L. Shluger, Adv. Mater. 23, 477 (2011).

61 L. Gross, F. Mohn, N. Moll, P. Liljeroth, and G. Meyer, Science 325, 1110 (2009).

62 R. Garcia and R. Perez, Surf. Sci. Rep. 47, 197 (2002).

${ }^{63}$ K. Voïtchovsky, J. J. Kuna, S. A. Contera, and E. Tosatti, Nature (2010).

64 M. Ricci, P. Spijker, F. Stellacci, J.-F. Molinari, and K. Voïtchovsky, Langmuir 29, 2207 (2013). 
65 C. Marutschke, D. Walters, J. Cleveland, I. Hermes, R. Bechstein, and A. Kühnle, Nanotechnology 25, 335703 (2014).

66 M. Ricci, P. Spijker, and K. Voïtchovsky, Nature Communications 5, (2014).

67 M. Ricci, J. J. Segura, B. Erickson, G. Fantner, F. Stellacci, and K. Voïtchovsky, Langmuir 27, 7563 (2015). 